\title{
A Millisecond Pulsar Progenitor to an Ultra-Compact Low-Mass X-ray Binary
}

\author{
S. C. Lundgren
}

Naval Research Lab, Code 7210, Washington, DC 20375

E. Ergma

Physics Dept., Tartu Univ., Ylikooli 18, EE2400 Tartu, Estonia

\section{J. M. Cordes}

Astronomy Department, Cornell University, Ithaca, NY 14853

\begin{abstract}
We discuss a twist in binary neutron star evolution scenarios in which a millisecond pulsar system is actually a progenitor for a lowmass X-ray binary system. After 7 billion years of orbital decay due to gravitational radiation, the millisecond pulsar PSR J0751+1807 will enter a low-mass X-ray binary phase. When the orbital period reaches about 5 minutes, the white dwarf will overflow its Roche lobe, transferring mass to the neutron star. Its predicted observational X-ray parameters are very similar to the ultra-compact low-mass X-ray binary $1820-303$. The ultimate fate of the system after Roche-lobe overflow is unknown. One possibility is that the companion may eventually tidally disrupt, leaving a disk of material which could form into planets.
\end{abstract}

\section{The Millisecond Pulsar Stage}

By extrapolating from current orbital and spin parameters for PSR J0751+1807 (Lundgren, Zepka, \& Cordes 1995) and assuming non-conservative mass transfer, a model for low-mass X-ray binary (LMXB) evolution (Ergma \& Sarna 1995) estimates an initial orbital period of one day, the first time the secondary overflowed its Roche-lobe. After the accretion ceased, the orbital period continued to decay due to gravitational radiation, with timescale (Landau \& Lifschitz 1959) $\tau_{g r}=(3 / 8) \mathrm{P}_{b} / \dot{\mathrm{P}}_{b} \approx 10^{9} \mathrm{yr} / M_{\mathrm{WD}}$. The orbital period decay $\left(\dot{\mathrm{P}}_{b}\right)$ can be measured for PSR J0751+1807 with a pulsar timing baseline of 10 years. The current upper limit on $\dot{\mathrm{P}}_{b}$ is 100 times the predicted value. From the orbital energy lost to gravitational radiation, the Roche-lobe radius, and the white dwarf (WD) mass-radius relation, we determined that the system will become an ultra-compact LMXB with $P_{b} \approx 5.45 \mathrm{~min}$ in 7 . Gyr, for $M_{\mathrm{WD}} \approx 0.15 M_{\odot}$.

The WD may be heated by the irradiation due to the millisecond pulsar (MSP), increasing the luminosity and temperature. The photospheric temperature of a companion star with the incoming energy thermalized in the photosphere is (Tavani 1992) $T \approx 1.5 \times 10^{4} K\left(\dot{\mathrm{P}}_{-20} I_{45} \mathrm{P}_{m s}^{-3}\right)^{1 / 4} a_{11}^{-1 / 2}$ with $a_{11}$ the 
orbital separation in units of $10^{11} \mathrm{~cm}$. Assuming favorable beaming in the PSR J0751+1807 system and if the WD age is close to the pulsar spindown time, the current optical luminosity of the WD is dominated by the effects of irradiation by the pulsar and will increase dramatically as the orbit decays.

\section{The Ultra-Compact LMXB Stage}

The mass-loss rate is determined by competition between WD expansion due to mass loss, orbital expansion due to mass transfer, and orbital decay due to gravitational radiation (Rappaport et al. 1987; Tutukov \& Yungelson 1979; Bisnovatyi-Kogan 1990). The initial mass-loss rate, $4 \times 10^{-7} M_{\odot} \mathrm{yr}^{-1}$, exceeds the Eddington accretion rate. Hence, the system becomes a bright LMXB with the Eddington luminosity. For stable mass transfer, the angular momentum carried away by mass lost from the system must be small, perhaps by ejection in jets (Bailyn \& Grindlay 1987). The jets must be maintained until the masstransfer rate drops below the Eddington accretion rate. When the accretion rates are favorable, PSR J0751+1807 may undergo an X-ray burster phase.

Since PSR J0751+1807 evolves through $\mathrm{P}_{b}=11 \mathrm{~min}$, it may be an evolutionary link to ultra-compact LMXBs like 1820-303 (Stella, Priedhorsky, \& White 1987). Confirming the $1820-303 / J 0751+1807$ connection will require more work to determine the survival probabilities for the WD in the initial Roche-lobe overflow stage of such an ultra-compact system.

Assuming the WD survives the super-Eddington mass transfer, the massloss rate drops to $10^{-9} M_{\odot} \mathrm{yr}^{-1}$ in $10^{7} \mathrm{yr}$, as the orbital period increases to $1000 \mathrm{~s}$ and the WD mass is depleted to $0.04 M_{\odot}$. Ruderman and Shaham (1985) calculated that a $0.04 M_{\odot}$ WD will tidally disrupt, ending the LMXB phase. The final fate of the system will be determined by the state of the mass remaining from the WD disruption. If the material is swept away by the pulsar beam, a solitary MSP would result. Alternatively, if a thick disk forms around the MSP, planets could form in the disk (van den Heuvel 1992).

The authors appreciated discussions with E.P.J. van den Heuvel, F. Verbunt, F. Camilo, and R. Foster about this work.

\section{References}

Bailyn, C. D. \& Grindlay, J. E. 1987, ApJ, 316, L25

Bisnovatyi-Kogan G.S., 1990a, Astrofyskia, 31, 567

Ergma E.\& Sarna M., 1995, MNRAS, in press

Landau L.D.\& Lifschitz E.M. 1959 "The classical theory of fields" Pergamon Press, Oxford

Lundgren, S. C., Zepka, A. F., \& Cordes, J. M. 1995, ApJ, 453, 419

Rappaport, S., Nelson, L. A., Ma, C. P., \& Joss, P. C. 1987, ApJ, 322, 842

Ruderman M.\&Shaham J., 1985, ApJ, 289, 244

Stella L., Priedhorsky W. \& White N.E.1987, ApJ, 318, L17

Tavani M., 1992, A\&A, 261, 472

Tutukov A.V.\&Yungelson L.R.,1979, Acta Astr., 29, 650

van den Heuvel, E.P.J. 1992, Nature, 356, 668 